\title{
NUMERICAL AND ASYMPTOTIC DESCRIPTION OF SOLITONS FOR GKdV EQUATIONS WITH NONHOMOGENEOUS NONLINEARITIES
}

\section{J. NOYOLA RODRIGUEZ and G. OMEL'YANOV}

Universidad de Sonora

Rosales y Encinas s/n, 83000

Hermosillo, Sonora

México

e-mail: jesnoyola89@gmail.com

omel@mat.uson.mx

\begin{abstract}
We consider a family of generalized KdV equations with a small dispersion and $C^{1}$-nonlinearity $g^{\prime}(u)$. We present sufficient conditions for $g^{\prime}(u)$ under which a soliton type solution exists and, moreover, pairs of solitary waves collide preserving in an asymptotic sense the KdV-type scenario of interaction. Furthermore, we create a finite difference scheme to simulate the solution of the Cauchy problem and present some numerical results for the interaction problem.
\end{abstract}

2010 Mathematics Subject Classification: 35Q53, 35D30, 65M12.

Keywords and phrases: GKDV equation, solitons, interaction, numerical simulation, finite differences scheme.

Received November 18, 2017

(ㄷ) 2017 Scientific Advances Publishers 


\section{Introduction}

We consider a generalization of the KdV equation of the form:

$$
\frac{\partial u}{\partial t}+\frac{\partial g^{\prime}(u)}{\partial x}+\varepsilon^{2} \frac{\partial^{3} u}{\partial x^{3}}=0, \quad x \in \mathbb{R}^{1}, \quad t>0,
$$

where $g^{\prime}(u) \stackrel{\text { def }}{=} \partial g / \partial u \in C^{1}$ is a real-valued function (for more detail see below) and $\varepsilon<<1$ is a small parameter. Such equations describe nonlinear wave phenomena in plasma physics. In particular, for some specific plasma states, ion-acoustic or dust-acoustic phenomena can be described by the KdV-type equation (1) with nonlinearities $g^{\prime}(u)=\alpha u^{3 / 2}+\beta u^{2}$ or $g^{\prime}(u)=\alpha u^{2}+\beta u^{3}, \alpha, \beta=$ const. $([8,11,12])$. To simplify the situation we restrict ourselves by non-negative $u$. Moreover, we assume that

$$
c_{1} u^{1+\delta_{1}} \leq g^{\prime}(u) \leq c_{2} u^{5-\delta_{2}}, \quad c_{i}=\text { const. }>0, \quad \delta_{i}=\text { const. }>0 .
$$

For homogeneous case $g^{\prime}(u)=u^{\kappa}, \kappa>1$, it is easy to find explicit solitary wave solutions (see below). Moreover, as it is well known nowadays, the solitons interact elastically in the integrable case $(\kappa=2$ and 3$)$. Almost the same is true for nonintegrable homogeneous case: the solitary waves interact elastically in the principal term in an asymptotic sense, whereas the nonintegrability implies the appearance of small radiation-type corrections [3-6], [9, 10]). At the same time, the existence of travelling wave solutions and the character of the solitary wave collision remains unknown for arbitrary nonlinearity. Our aim is to consider these open problems.

The contents of the paper is the following: in Section 2, we find a class of nonlinearities which admits soliton type solutions, in Section 3, we demonstrate the elastic (in the leading asymptotic term) scenario of two soliton collision, and in the last section, we describe a finite difference scheme for this equation. 


\section{Solitary Wave Solution}

Before the search of admissible nonlinearities we should determine the type of solitary waves which will be under consideration.

Definition 1. A function

$$
u=A \omega(\beta(x-V t) / \varepsilon, A)
$$

is called "soliton type solitary wave" if $\omega(\eta, \cdot) \in \mathcal{C}^{\infty}\left(\mathcal{R}^{1}\right)$ is an even function such that $\omega(0, \cdot)=1, \omega(\eta, \cdot)<1$ for $\eta \neq 0$, and $\partial^{2} \omega /\left.\partial \eta^{2}\right|_{\eta=0}<0$.

Moreover, we assume that

$$
\omega(\eta, \cdot) \rightarrow 0 \quad \text { as } \quad \eta \rightarrow \pm \infty
$$

with an exponential rate. Next, we suppose that $\beta=\beta(A), V=V(A)$, $\omega=\omega(\cdot, A)$ are $C^{1}$-functions uniformly in the parameter $A>0$, and

$$
\partial \omega(\eta, A) / \partial A \rightarrow 0 \quad \text { as } \quad \eta \rightarrow 0 \quad \text { or } \quad \eta \rightarrow \pm \infty .
$$

Theorem 1. Let $g(u) \in \mathcal{C}^{2}(u \geq 0) \bigcap \mathcal{C}^{\infty}(u>0)$ satisfy (2) and be such that

$$
g(u)=u^{2} g_{1}(u)
$$

where the Hölder continuous function $g_{1}$ satisfies the conditions:

$$
g_{1}(0)=0, \quad g_{1}(u)>0 \quad \text { and } \quad g_{1}^{\prime}(u)>0 \quad \text { for } u>0 .
$$

Then the Equation (1) has a soliton type solitary wave solution.

Proof. Let us substitute the desired form (3) into the Equation (1) and integrate it using the condition (4). We obtain

$$
\frac{\beta^{2}}{V}\left(\frac{d \omega}{d \eta}\right)^{2}=\omega^{2}\left(1-\frac{2 g_{1}(A)}{V} G(\omega, A)\right), G(\omega, A) \stackrel{\text { def }}{=} g_{1}(A \omega) / g_{1}(A) .
$$

Next, $\omega$ can be a smooth even function if and only if $\partial \omega /\left.\partial \eta\right|_{\eta=0}=0$. This and the normalization condition $\omega(0, \cdot)=1$ imply the equality 


$$
V=2 g_{1}(A)
$$

In order to simplify the equation, we define the free parameter $\beta$,

$$
\beta^{2}=V
$$

and obtain the final version of the equation for $\omega$

$$
\frac{d \omega}{d \eta}= \pm \omega \sqrt{1-G(\omega, A)}
$$

where the sign should be - for $\eta>0$ and + for $\eta<0$.

To complete the proof it is enough to analyze the implicit representation of $\omega$ which corresponds to (11).

Example. The function

$$
g_{1}(z)=\sum_{k=1}^{n} c_{k} z^{q_{k}}, \quad \delta_{1} \leq q_{1}<q_{2}<\cdots<q_{n}<4, \quad c_{k}>0,
$$

satisfies the conditions (7). If $n=1$, then the solution of (11) does not depend on $A$ and has the form:

$$
\omega(\eta)=\left\{\cosh \left(q_{1} \eta / 2\right)\right\}^{-2 / q_{1}}, \quad V=2 c_{1} A^{q_{1}} .
$$

\section{Two-soliton Asymptotic Solution}

\subsection{Main definitions}

Obviously, there is not any hope to find both the exact multi-soliton solution to (1) and an asymptotics in the classical sense. So, we will construct a weak asymptotic solution. The weak asymptotics method (see, e.g., [1-7], [9, 10] and references therein) takes into account the fact that soliton-type solutions which are smooth for $\varepsilon>0$ become non-smooth in the limit as $\varepsilon \rightarrow 0$. Thus, it is possible to treat such solutions as a mapping $\mathcal{C}^{\infty}\left(0, T ; \mathcal{C}^{\infty}\left(\mathbb{R}_{x}^{1}\right)\right) \quad$ for $\quad \varepsilon=$ const. $>0$ and only as $\mathcal{C}\left(0, T ; \mathcal{D}^{\prime}\left(\mathbb{R}_{x}^{1}\right)\right)$ 
uniformly in $\varepsilon \geq 0$. Accordingly, the remainder should be small in the weak sense. The main advantage of the method is such that we can ignore the real shape of the colliding waves but look for (and find) exceptionally their main characteristics. For the solitons, they are the amplitudes and trajectories of the waves.

Similarly the famous Whitham method we define a weak asymptotic solution as a function which satisfies (in a weak sense) some conservation laws, in fact two laws for the two-phase asymptotics. For the Equation (1), let us write the first conservation laws in the differential form:

$$
\frac{\partial Q_{j}}{\partial t}+\frac{\partial P_{j}}{\partial x}=\varepsilon^{2} \frac{\partial^{3} R_{j}}{\partial x^{3}}, \quad j=1,2
$$

where the first one is the Equation (1) the same, namely,

$$
\begin{gathered}
Q_{1}=u, \quad P_{1}=g^{\prime}(u), \quad Q_{2}=u^{2}, \quad P_{2}=-2 g_{2}(u)-3\left(\varepsilon u_{x}\right)^{2}, \\
R_{1}=u, \quad R_{2}=u^{2}, \quad g_{2}(u)=g(u)-u g^{\prime}(u) .
\end{gathered}
$$

Next, we define the smallness in the weak sense:

Definition 2. A function $v(t, x, \varepsilon)$ is said to be of the value $O_{\mathcal{D}^{\prime}}\left(\varepsilon^{k}\right)$ if the relation

$$
\int_{-\infty}^{\infty} v(t, x, \varepsilon) \psi(x) d x=O\left(\varepsilon^{k}\right)
$$

holds uniformly in $t$ for any test function $\psi \in \mathcal{D}\left(\mathbb{R}_{x}^{1}\right)$. The right-hand side here is a $\mathcal{C}^{\infty}$-function for $\varepsilon=$ const. $>0$ and a piecewise continuous function uniformly in $\varepsilon \geq 0$.

As it has been demonstrated in [3, 9], the correct definition of twosoliton asymptotics is the following: 
Definition 3. A sequence $u(t, x, \varepsilon)$, belonging to $\mathcal{C}^{\infty}\left(0, T ; \mathcal{C}^{\infty}\left(\mathbb{R}_{x}^{1}\right)\right)$ for $\varepsilon=$ const. $>0$ and belonging to $\mathcal{C}\left(0, T ; \mathcal{D}^{\prime}\left(\mathbb{R}_{x}^{1}\right)\right)$ uniformly in $\varepsilon$, is called a weak asymptotic mod $O_{\mathcal{D}^{\prime}}\left(\varepsilon^{2}\right)$ solution of (1) if the relations (14) hold uniformly in $t$ with the accuracy $O_{\mathcal{D}^{\prime}}\left(\varepsilon^{2}\right)$.

Let us consider the interaction of two solitary waves for the model (1) with the initial data

$$
\left.u\right|_{t=0}=\sum_{i=1}^{2} A_{i} \omega\left(\beta_{i} \frac{x-x_{i}^{0}}{\varepsilon}, A_{i}\right)
$$

where $A_{2}>A_{1}>0, x_{1}^{0}-x_{2}^{0}=$ const. $>0$ and we assume the same relations between $A_{i}, \beta_{i}$ and $V_{i}$ as in (9), (10). Obviously, the trajectories $x=V_{i} t+x_{i}^{0}$ have a joint point $x=x^{*}$ at a time instant $t=t^{*}$

Following [3, 9], we write the asymptotic ansatz in the form:

$$
u=\sum_{i=1}^{2} G_{i}(\tau) \omega\left(\beta_{i} \frac{x-\varphi_{i}(t, \tau, \varepsilon)}{\varepsilon}, A_{i}\right), \quad G_{i}(\tau)=A_{i}+S_{i}(\tau)
$$

Here $\varphi_{i}=\varphi_{i 0}(t)+\varepsilon \varphi_{i 1}(\tau)$, where $\varphi_{i 0}=V_{i} t+x_{i}^{0}$, are the trajectories of noninteracting solitary waves;

$$
\tau=\psi_{0}(t) / \varepsilon, \quad \psi_{0}(t)=\beta_{1}\left(\varphi_{20}(t)-\varphi_{10}(t)\right)
$$

denotes the "fast time"; the phase and amplitude corrections $\varphi_{i 1}, S_{i}$ are smooth functions such that

$$
\begin{gathered}
\varphi_{i 1}(\tau) \rightarrow 0 \text { as } \tau \rightarrow-\infty, \quad \varphi_{i 1}(\tau) \rightarrow \varphi_{i 1}^{\infty}=\text { const }_{i} \text { as } \tau \rightarrow+\infty, \\
S_{i}(\tau) \rightarrow 0 \text { as } \tau \rightarrow \pm \infty
\end{gathered}
$$

with exponential rates. 


\subsection{Asymptotic construction}

To construct the asymptotics, we should calculate the weak expansions of the terms from the left-hand sides of the relations (14). It is easy to check that

$$
u=\varepsilon \sum_{i=1}^{2} a_{1, i} \frac{G_{i}}{\beta_{i}} \delta\left(x-\varphi_{i}\right)+O_{\mathcal{D}^{\prime}}\left(\varepsilon^{3}\right),
$$

where $\delta(x)$ is the Dirac delta-function. Here and in what follows we use the notation

$$
a_{k, i} \stackrel{\operatorname{def}}{=} \int_{-\infty}^{\infty}\left(\omega\left(\eta, A_{i}\right)\right)^{k} d \eta, \quad k>0, \quad a_{2, i}^{\prime} \stackrel{\operatorname{def}}{=} \int_{-\infty}^{\infty}\left(\omega^{\prime}\left(\eta, A_{i}\right)\right)^{2} d \eta .
$$

At the same time for any $F(u) \in C^{1}$

$$
\begin{aligned}
& \int_{-\infty}^{\infty} F\left(\sum_{i=1}^{2} G_{i} \omega\left(\beta_{i} \frac{x-\varphi_{i}}{\varepsilon}, A_{i}\right)\right) \psi(x) d x \\
& =\varepsilon \sum_{i=1}^{2} \frac{1}{\beta_{i}} \int_{-\infty}^{\infty} F\left(A_{i} \omega\left(\eta, A_{i}\right)\right) \psi\left(\varphi_{i}+\varepsilon \frac{\eta}{\beta_{i}}\right) d \eta+\frac{\varepsilon}{\beta_{2}} \int_{-\infty}^{\infty}\left\{F \left(G_{1} w\left(\eta_{12}, A_{1}\right)\right.\right. \\
& \left.\left.\quad+G_{2} \omega\left(\eta, A_{2}\right)\right)-F\left(A_{1} \omega\left(\eta_{12}, A_{1}\right)\right)-F\left(A_{2} \omega\left(\eta, A_{2}\right)\right)\right\} \psi\left(\varphi_{2}+\varepsilon \frac{\eta}{\beta_{2}}\right) d \eta,
\end{aligned}
$$

where

$$
\left.\eta_{12}=\theta \eta-\sigma, \quad \sigma=\beta_{1}\left(\varphi_{1}-\varphi_{2}\right)\right) / \varepsilon, \quad \theta=\beta_{1} / \beta_{2} .
$$

We take into account that the second integrand in the right-hand side of (23) vanishes exponentially fast as $\left|\varphi_{1}-\varphi_{2}\right|$ grows, thus, its main contribution is at the point $x^{*}$. We write

$$
\varphi_{i 0}=x^{*}+V_{i}\left(t-t^{*}\right)=x^{*}+\varepsilon \frac{V_{i}}{\psi_{0}} \tau \quad \text { and } \quad \varphi_{i}=x^{*}+\varepsilon \chi_{i}
$$

where $\dot{\varphi}_{0}=\beta_{1}\left(V_{2}-V_{1}\right), \chi_{i}=V_{i} \tau / \dot{\psi}_{0}+\varphi_{i 1}$. It remains to apply the formula 


$$
f(\tau) \delta\left(x-\varphi_{i}\right)=f(\tau) \delta\left(x-x^{*}\right)-\varepsilon \chi_{i} f(\tau) \delta^{\prime}\left(x-x^{*}\right)+O_{\mathcal{D}^{\prime}}\left(\varepsilon^{2}\right),
$$

which holds for each $\varphi_{i}$ of the form (25) with slowly increasing $\chi_{i}$ and for $f(\tau)$ from the Schwartz space. Moreover, the second term in the righthand side of (26) is $O_{\mathcal{D}^{\prime}}(\varepsilon)$. Thus, under the assumptions (19) and (20), we obtain the weak asymptotic expansion of $F(u)$ in the final form:

$$
F(u)=\varepsilon \sum_{i=1}^{2} F\left(A_{i}\right) \frac{a_{F, i}}{\beta_{i}} \delta\left(x-\varphi_{i}\right)+\varepsilon \frac{F\left(A_{2}\right)}{\beta_{2}} \Re_{F} \delta\left(x-x^{*}\right)+O_{\mathcal{D}^{\prime}}\left(\varepsilon^{2}\right),
$$

where

$$
\begin{gathered}
a_{F, i}=F\left(A_{i}\right)^{-1} \int_{-\infty}^{\infty} F\left(A_{i} \omega\left(\eta, A_{i}\right)\right) d \eta \\
\mathfrak{R}_{F}=F\left(A_{2}\right)^{-1} \int_{-\infty}^{\infty}\left\{F\left(\sum_{i=1}^{2} G_{i} \omega\left(\eta_{i 2}, A_{i}\right)\right)-\sum_{i=1}^{2} F\left(A_{i} \omega\left(\eta_{i 2}, A_{i}\right)\right)\right\} d \eta
\end{gathered}
$$

and $\eta_{22} \stackrel{\text { def }}{=} \eta$. Next calculating weak expansions for other terms from (14) we pass to linear combinations of $\delta^{\prime}\left(x-\varphi_{i}\right), i=1,2, \delta\left(x-x^{*}\right)$, and $\delta^{\prime}\left(x-x^{*}\right)$ (see also $\left.[3,4,9]\right)$. Therefore, we obtain:

$$
\begin{gathered}
a_{1, i} V_{i} K_{i 0}^{(1)}-a_{g^{\prime}, i} g^{\prime}\left(A_{i}\right) / \beta_{i}=0, \quad i=1,2 \\
a_{2, i} V_{i} K_{i 0}^{(2)}+2 a_{g_{2}, i} g_{2}\left(A_{i}\right) / \beta_{i}+3 a_{2, i}^{\prime} \beta_{i}^{2} K_{i 0}^{(2)}=0, \quad i=1,2 \\
\sum_{i=1}^{2} a_{1, i} K_{i 1}^{(1)}=0, \quad \sum_{i=1}^{2} a_{2, i} K_{i 1}^{(2)}+2 \widetilde{a}_{2} \frac{G_{1} G_{2}}{\beta_{2}} R_{2}^{(0)}=0, \quad i=1,2 \\
\dot{\varphi}_{0} \frac{d}{d \tau} \sum_{i=1}^{2} a_{1, i}\left\{K_{i 0}^{(1)} \varphi_{i 1}+\chi_{i} K_{i 1}^{(1)}\right\}=f \\
\dot{\varphi}_{0} \frac{d}{d \tau}\left\{\sum_{i=1}^{2} a_{2, i}\left(K_{i 0}^{(2)} \varphi_{i 1}+\chi_{i} K_{i 1}^{(2)}\right)+2 \frac{\tilde{a}_{2}}{\beta_{2}} G_{1} G_{2}\left(\chi_{2} R_{2}^{(0)}+\frac{1}{\beta_{2}} R_{2}^{(1)}\right)\right\}=F
\end{gathered}
$$


where

$$
\begin{gathered}
f=\frac{g^{\prime}\left(A_{2}\right)}{\beta_{2}} \Re_{g^{\prime}}, \quad \widetilde{a}_{2}^{\prime}=\sqrt{a_{2,1}^{\prime} a_{2,2}^{\prime}}, \\
F=-2 \frac{g_{2}\left(A_{2}\right)}{\beta_{2}} \mathfrak{R}_{g_{2}}-3\left\{\sum_{i=1}^{2} a_{2, i}^{\prime} \beta_{i}^{2} K_{i 1}^{(2)}+2 \widetilde{a}_{2}^{\prime} \beta_{1} G_{1} G_{2} R_{2,1}^{(0)}\right\}, \\
K_{i}^{(n)}=\frac{G_{i}^{n}}{\beta_{i}}, \quad K_{i 0}^{(n)}=\frac{A_{i}^{n}}{\beta_{i}}, \quad K_{i 1}^{(n)}=K_{i}^{(n)}-K_{i 0}^{(n)}, \\
R_{2}^{(i)}=\frac{1}{\widetilde{a}_{2}} \int_{-\infty}^{\infty} \eta^{i} \omega\left(\eta_{12}, A_{1}\right) \omega\left(\eta, A_{2}\right) d \eta, \widetilde{a}_{2}=\sqrt{a_{2,1} a_{2,2}},
\end{gathered}
$$

and $R_{2,1}^{(0)}$ is of the form (37) for $R_{2}^{(0)}$ but with $\widetilde{a}_{2}^{\prime}$, $\omega^{\prime}$ instead of $\widetilde{a}_{2}$, $\omega$.

\subsection{Analysis of the model equations (30)-(33)}

Analyzing the Equation (11) it is easy to verify the statement (see also [9]):

Lemma 1. The algebraic equations (30), (31) imply again the relations (9), (10) between $A_{i}, \beta_{i}$, and $V_{i}$.

To analyze the system (32), we assume:

$$
A_{i}>1, \quad i=1,2, \quad \theta<<1 .
$$

Moreover, let the function $g_{1}$ be of the form (12). Then (9), (10) imply:

$$
A_{i}=c^{\prime} \beta_{i}^{q^{\prime}}\left(1+O\left(A_{i}^{q_{n-1}-q_{n}}\right)\right), \quad q^{\prime}=2 / q_{n}, c^{\prime}=\left(2 c_{n}\right)^{-1 / q_{n}}, i=1,2 .
$$

Let us define the following notation:

$$
\kappa_{i}=\frac{S_{i}}{\beta_{i}}\left(c^{\prime} \beta_{2}^{q^{\prime}-1}\right)^{-1}, \quad i=1,2 .
$$


Definition 4. A function $f(\tau, \theta)$ is said to be of the value $O_{S}\left(\theta^{k}\right)$ if there exists a function $s(\tau)$ from the Schwartz space such that the estimate

$$
|f(\tau, \theta)| \leq c \theta^{k}|s(\tau)|
$$

holds uniformly in $\tau$ for a constant $c>0$.

We also note that, under the conditions (19), the convolution $R_{2}^{(0)} \rightarrow 0$ as $\tau \rightarrow \pm \infty$ with an exponential rate.

Assumptions (38) and (12) allow us to prove the statement:

Lemma 2. Let the assumptions (12) and (38) be satisfied. Then the algebraic equations (32) have a unique solution $S_{1}, S_{2}$ with the property (20). Moreover,

$$
\kappa_{1}=\frac{\sqrt{\bar{a}_{2}}}{\bar{a}_{1}} \theta^{q^{\prime}} R_{2}^{(0)}+O_{S}\left(\theta^{2}+\theta^{2 q^{\prime}}\right), \quad \kappa_{2}=-\bar{a}_{1} \kappa_{1}, \quad \bar{a}_{i}=a_{i, 1} / a_{i, 2} .
$$

Now let us simplify the Equations (33). Firstly we note that in view of the first part of Equation (32) and the identity $\beta_{1}\left(\chi_{1}-\chi_{2}\right)=\sigma$ one can eliminate $\chi_{i}$ from the left-hand side of the first part of Equation (33), since

$$
\sum_{i=1}^{2} a_{1, i}\left\{K_{i 0}^{(1)} \varphi_{i 1}+\chi_{i} K_{i 1}^{(1)}\right\}=\sum_{i=1}^{2} a_{1, i} K_{i 0}^{(1)} \varphi_{i 1}+a_{1,1} \frac{\sigma}{\beta_{1}} K_{11}^{(1)} .
$$

Simplifying in the same manner the second part of Equation (33), we transform this system to the following form:

$$
\begin{gathered}
\dot{\varphi}_{0} \frac{d}{d \tau}\left\{\sum_{i=1}^{2} a_{1, i} K_{i 0}^{(1)} \varphi_{i 1}+a_{1,1} \frac{\sigma}{\beta_{1}} K_{11}^{(1)}\right\}=f, \\
\dot{\psi}_{0} \frac{d}{d \tau}\left\{\sum_{i=1}^{2} a_{2, i} K_{i 0}^{(2)} \varphi_{i 1}+a_{2,1} \frac{\sigma}{\beta_{1}} K_{11}^{(2)}+2 \widetilde{a}_{2} \theta K_{1}^{(1)} K_{2}^{(1)} R_{2}^{(1)}\right\}=F,
\end{gathered}
$$

where $f$ and $F$ are defined in (34) and (35). 
The second step is the elimination of $\varphi_{i 1}$ from the model system. To do it we divide $\sigma$ into the growing $\beta_{1}\left(V_{1}-V_{2}\right) \tau / \dot{\psi}_{0}=-\tau$ and the bounded (if the assumptions (19) are satisfied) $\tilde{\sigma}=\sigma+\tau$ parts. Since

$$
\varphi_{11}=\varphi_{21}+\tilde{\sigma} / \beta_{1}
$$

we obtain from (43)

$$
\dot{\varphi}_{0} \frac{d}{d \tau}\left\{r_{1} \varphi_{21}+\frac{\sigma}{\beta_{1}} K_{1}^{(1)}\right\}=\frac{f}{a_{1,1}}-\frac{\dot{\varphi}_{0}}{\beta_{1}} K_{10}^{(1)} .
$$

Here and in what follows we use the notation

$$
r_{j}=\sum_{i=1}^{2} \frac{a_{j, i}}{a_{j, 1}} K_{i 0}^{(j)}, \quad \text { for } \quad j=1,2 .
$$

Now, transforming (44) in the same manner and applying the first part of assumption (19) we pass to the problem:

$$
\frac{d}{d \tau} Q(\sigma)=\mathfrak{F}(\sigma),\left.\quad \frac{\sigma}{\tau}\right|_{\tau \rightarrow-\infty} \rightarrow-1
$$

where

$$
\begin{gathered}
Q=\frac{\sigma}{\beta_{1}}\left\{K_{1}^{(2)}-\frac{r_{2}}{r_{1}} K_{1}^{(1)}\right\}+\frac{2}{\sqrt{\bar{a}_{2}}} K_{1}^{(1)} K_{2}^{(1)} R_{2}^{(1)}, \\
\mathfrak{F}=-\frac{1}{\beta_{1}}\left\{K_{10}^{(2)}-\frac{r_{2}}{r_{1}} K_{10}^{(1)}\right\}+\frac{1}{\dot{\psi}_{0}}\left\{\frac{1}{a_{2,1}} F-\frac{r_{2}}{a_{1,1} r_{1}} f\right\} .
\end{gathered}
$$

Sufficiently simple analysis of the Equation (48) implies the statement:

Lemma 3. Under the assumptions (12), (38), $Q(\sigma) \in C^{2}\left(\mathbb{R}^{1}\right)$ and $\mathfrak{F}(\sigma) \in C^{1}\left(\mathbb{R}^{1}\right)$. Moreover, the following relations hold:

$\frac{d Q}{d \sigma}=-\frac{A_{1} A_{2}}{\beta_{1}^{2}}\left\{\frac{\bar{a}_{2}}{\bar{a}_{1}}-\theta^{q^{\prime}}+O_{S}\left(\theta+\theta^{q^{\prime}}\right)\right\}, \mathfrak{F}=\frac{A_{1} A_{2}}{\beta_{1}^{2}}\left\{\frac{\bar{a}_{2}}{\bar{a}_{1}}-\theta^{q^{\prime}}+O_{S}\left(\theta+\theta^{q^{\prime}}\right)\right\}$. 
The uniform in $\tau$ inequality $\mathfrak{F}>0$ and the exponential type behaviour of $\mathfrak{F}$ and $Q$ imply the existence of the function $\sigma$ such that $\tilde{\sigma}=\sigma+\tau$ is bounded and tends to its limiting values with an exponential rate. This and the equalities (45), (46) justify the existence of the required phase corrections $\varphi_{i 1}$ with the property (19).

The main result of this section is the following:

Theorem 2. Let the assumptions (12), (38) be satisfied. Then the solitary wave collision in the problem (1), (17) preserves the elastic scenario with accuracy $O_{\mathcal{D}^{\prime}}\left(\varepsilon^{2}\right)$ in the sense of Definition 2. The weak asymptotic solution has the form (18).

The next theorem allows us to treat the weak asymptotics (30) in the classical sense:

Theorem 3. Let the assumptions (12), (38) be satisfied. Then the function $u$ of the form (18) is a weak asymptotic $\bmod O_{\mathcal{D}^{\prime}}\left(\varepsilon^{2}\right)$ solution of (1) if and only if $u$ satisfies the following conservation and balance laws:

$$
\begin{gathered}
\frac{d}{d t} \int_{-\infty}^{\infty} u d x=0, \frac{d}{d t} \int_{-\infty}^{\infty} u^{2} d x=0, \frac{d}{d t} \int_{-\infty}^{\infty} x u d x-\int_{-\infty}^{\infty} g^{\prime}(u) d x=0 \\
\frac{d}{d t} \int_{-\infty}^{\infty} x u^{2} d x+2 \int_{-\infty}^{\infty} g_{2}(u) d x+3 \int_{-\infty}^{\infty}\left(\varepsilon \frac{\partial u}{\partial x}\right)^{2} d x=0
\end{gathered}
$$

To prove the Theorem 3, it is enough to rewrite the equalities (30)-(33) as integrals of the function (18) and its derivatives.

Results of direct numerical simulations confirm the traced asymptotic analysis. Figure 1 depicts the collision of two solitons for the nonlinearity $g^{\prime}=u^{3 / 2}+u^{2}$ in the case $\varepsilon=0.1$ (see also [10] for the nonlinearity $\left.u^{3 / 2}\right)$. 


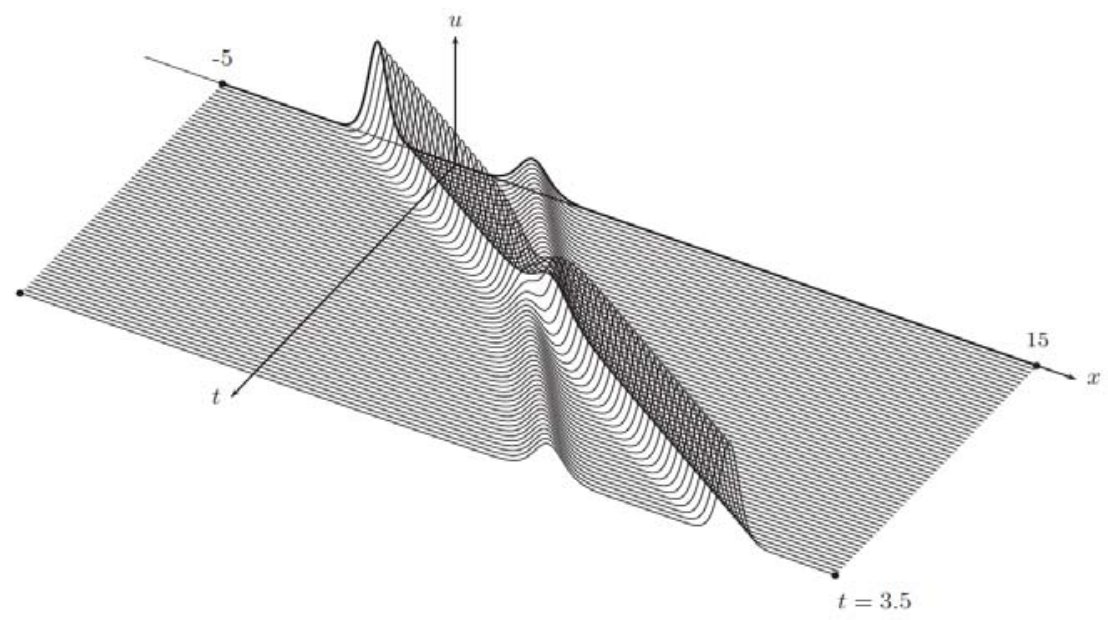

Figure 1. Evolution of two solitary waves with $A_{1}=1.5$ and $A_{2}=0.5$ for the nonlinearity $g^{\prime}=u^{3 / 2}+u^{2}$.

\section{Finite Difference Scheme}

Obviously, it is impossible to create any finite difference scheme for the problem (1), (17) which remains stable uniformly in $\varepsilon \rightarrow 0$ and $t \in(0, T], T=$ const. So we will treat $\varepsilon$ as a small but fixed constant.

Concerning the original KdV-type equation (1), we simulate firstly the Cauchy problem by a mixed problem over a $\Omega_{T}=\{x \in(0, L)$, $t \in(0, T], L=$ const. $\}$ with zero boundary value. Next, we define a mesh $\Omega_{T, \tau, h}=\left\{\left(x_{i}, t_{j}\right) \stackrel{\text { def }}{=}(i h, j \tau), i=0, \ldots, N, j=0, \ldots, J\right\}$. To simulate the interaction phenomena, we assume that $L, T$, and the initial front positions $x_{i}^{0}$ of solitons are such that the solitary wave trajectories have an intersection point which belongs to $(0, L) \times(0, T / 2)$. Furthermore, we assume that, uniformly in $t \leq T$,

$$
\left.|u(x, t)|_{x \in[0, \delta]}\left|\leq c \max \left\{\varepsilon^{2}, h^{2}\right\}, \quad\right| u(x, t)\right|_{x \in[L-\delta, L]} \mid \leq c \max \left\{\varepsilon^{2}, h^{2}\right\},
$$

for some $c>0$ and sufficiently small $\delta>0$. 
To create a finite difference scheme for the Equation (1), we should choose appropriate approximations for the differential terms and for the nonlinear term. Let us do it separately. We write firstly a preliminary nonlinear "scheme" of the local accuracy $O\left(\tau+h^{2}\right)$ :

$$
y_{\bar{t}}+Q(y)+\varepsilon^{2} \gamma y_{x \bar{x} \dot{x}}+\varepsilon^{2} \nu h y_{x \bar{x} x}=0
$$

where $y \stackrel{\text { def }}{=} y_{i}^{j} \stackrel{\text { def }}{=} u\left(x_{i}, t_{j}\right), \gamma=1-h \nu, \nu>0$ is a constant,

$$
y_{i x}^{j} \stackrel{\text { def }}{=} \frac{y_{i+1}^{j}-y_{i}^{j}}{h} \stackrel{\text { def }}{=} y_{x}, y_{i \bar{x}}^{j} \stackrel{\text { def }}{=} \frac{y_{i}^{j}-y_{i-1}^{j}}{h} \stackrel{\text { def }}{=} y_{\bar{x}}, y_{i \bar{t}}^{j} \stackrel{\text { def }}{=} \frac{y_{i}^{j}-y_{i}^{j-1}}{\tau} \stackrel{\text { def }}{=} y_{\bar{t}} \text {, }
$$

and $y_{\dot{x}} \stackrel{\text { def }}{=}\left(y_{x}+y_{\bar{x}}\right) / 2$.

For the nonlinearity of the form (6), (12), we define

$$
Q(y)=\sum_{k=1}^{n} c_{k}\left(q_{k}+1\right) Q_{k}(y), \quad Q_{k}(y)=\left\{\left(y^{q_{k}+1}\right)_{\dot{x}}+y^{q_{k}} y_{\dot{x}}\right\} .
$$

This choice of $Q$ guarantees the equality

$$
h \sum_{i=1}^{N-1} y_{i}^{j} Q\left(y_{i}^{j}\right)=0
$$

for all set functions $y$ with zero boundary value. This implies the identity

$$
\partial_{\bar{t}}\left\|y^{j}\right\|^{2}+\tau\left\|y_{\bar{t}}^{j}\right\|^{2}+\nu h^{2}\left\|\varepsilon y_{x \bar{x}}^{j}\right\|^{2}=0,
$$

where $\|\cdot\|$ is the discrete version of the $L^{2}(0, L)$ norm. Obviously, the equality (54) is the discrete version of the conservation law

$$
\frac{d}{d t} \int_{-\infty}^{\infty} u^{2} d x=0
$$

The additional terms in (54) demonstrate the property of a parabolic-type regularization of the scheme (52). On the other hand, (54) implies a weak convergence of $y_{i}^{j}$ as $\tau, h \rightarrow 0$ to the solution of the corresponded mixed problem for (1). 
Theorem 4. Let the systems (52), (53) supplemented by initial and boundary conditions have a solution $y=y_{\tau, h}$ and let $y_{\tau, h}(x, t)$ be its continuation over $\Omega_{T}$. Then there exists a subsequence $y_{\bar{\tau}, \bar{h}}(x, t)$ such that

$$
y_{\bar{\tau}, \bar{h}} \rightarrow u \quad \text { *-weakly in } L^{\infty}\left((0, T) ; L^{2}(0, L)\right) \bigcap L^{2}\left((0, T) ; H_{0}^{1}(0, L)\right)
$$

as $\tau, h \rightarrow 0$.

Furthermore, we note that for polynomial $g^{\prime}$, that is for integer $q_{k}$,

$$
g^{\prime}(u)=\sum_{k=1}^{3} c_{k}(k+2) u^{k+1},
$$

it should be used a more effective formula [5]:

$$
Q(y)=2 \sum_{k=1}^{3} c_{k} Q_{k}(y), \quad Q_{k}(y)=\sum_{l=1}^{k+1} y^{k+1-l}\left(y^{l}\right)_{\dot{x}} .
$$

Such approximation implies that the solution of (52) satisfies both (54) and the mean value conservation law:

$$
\partial_{\bar{t}} \sum_{i=0}^{N} y_{i}^{j}=0, \quad j=1,2, \ldots
$$

Moreover, for the KdV case, $g^{\prime}=u^{2}$, and sufficiently smooth initial data it can be proved the following a-priori estimate:

$$
\left\|\left(\varepsilon \partial_{x}\right)^{k} y^{j}\right\|^{2}+\tau\left\|\left|\left(\varepsilon \partial_{x}\right)^{k} y_{\bar{t}}\right|\right\|^{2}(j)+h^{2}\left\|\left|\left(\varepsilon \partial_{x}\right)^{k} \varepsilon y_{x \bar{x}}\right|\right\|^{2}(j) \leq C_{k},
$$

where $k \geq 1,\||\cdot|\|(j)$ is the discrete version of the $L^{2}\left(\Omega_{t_{j}}\right)$ norm, and $C_{k}=C_{k}\left(\left\|y^{0}\right\|, \ldots,\left\|\left(\varepsilon \partial_{x}\right)^{k} y^{0}\right\|, \varepsilon\right)$ does not depend on $\tau$ and $h$. Obviously, the estimate (59) implies the convergence of a subsequence of $y$. 
Theorem 5. Let the assumptions of Theorem 4 be satisfied and $g^{\prime}=u^{2}$. Then there exists a subsequence $y_{\bar{\tau}, \bar{h}}(x, t)$ such that

$$
\begin{aligned}
& y_{\bar{\tau}, \bar{h}} \rightarrow u \quad \text { *-weakly in } \quad L^{\infty}\left((0, T) ; H_{0}^{4}(0, L)\right), \\
& \frac{\partial y_{\bar{\tau}, \bar{h}}}{\partial t} \rightarrow \frac{\partial u}{\partial t} \quad \text { *-weakly in } \quad L^{\infty}\left((0, T) ; H_{0}^{1}(0, L)\right)
\end{aligned}
$$

as $\tau, h \rightarrow 0$.

To solve the Equation (52) for any fixed $j \geq 1$ we apply an iterative procedure. Namely, we construct a sequence of functions $\varphi(s) \stackrel{\text { def }}{=}\left\{\varphi_{0}(s)\right.$, $\left.\ldots, \varphi_{N}(s)\right\}, s \geq 0$, where $\varphi(0)=\check{y} \stackrel{\text { def }}{=} y^{j-1}$ and the consequent terms $\varphi(s)$ we define depending on the nonlinearity. If $q_{1}<1$, then to find $\varphi(s)$ we solve the linear system:

$$
\varphi+\tau R(\bar{\varphi}, \varphi)+\tau \varepsilon^{2} \gamma \varphi_{x \bar{x} \dot{x}}+\tau \varepsilon^{2} \nu h \varphi_{x \bar{x} x}=\check{y},
$$

where $\varphi=\varphi(s), \bar{\varphi}=\varphi(s-1)$,

$$
R(\bar{\varphi}, \varphi)=\sum_{k=1}^{n} c_{k}\left(q_{k}+1\right) R_{k}(\bar{\varphi}, \varphi), \quad R_{k}(\bar{\varphi}, \varphi)=\left(\bar{\varphi}^{q_{k}} \varphi\right) \dot{x}+\bar{\varphi}^{q_{k}} \varphi_{\dot{x}}
$$

If $q_{1} \geq 1$, then $\varphi(s)$ should satisfy the following system:

$$
\varphi+\tau \sum_{k=1}^{n} c_{k}\left(q_{k}+1\right) R_{k}(\bar{\varphi}, w)+\tau \varepsilon^{2} \gamma \varphi_{x \bar{x} \dot{x}}+\tau \varepsilon^{2} \nu h \varphi_{x \bar{x} x}=\breve{y}-\tau Q(\bar{\varphi}),
$$

where $w=\varphi-\bar{\varphi}, Q(y)$ has been defined in (53), and

$$
R_{k}(\bar{\varphi}, w)=\left(q_{k}+1\right)\left(\bar{\varphi}^{q_{k}} w\right)_{\dot{x}}+\bar{\varphi}^{q_{k}} w_{\dot{x}}+q_{k} \bar{\varphi}^{q_{k}-1} \bar{\varphi}_{\dot{x}} w .
$$

Finally, for polynomial nonlinearity of the form (56), we consider again the equations similar to (63) but with others $Q$ and $R_{k}$ : 


$$
\varphi+2 \tau \sum_{k=1}^{n} c_{k} R_{k}(\bar{\varphi}, w)+\tau \varepsilon^{2} \gamma \varphi_{x \bar{x} \dot{x}}+\tau \varepsilon^{2} \nu h \varphi_{x \tilde{x} x}=\check{y}-\tau Q(\bar{\varphi})
$$

where $Q(y)$ has been defined in (57), and

$$
R_{k}(\bar{\varphi}, w)=\sum_{l=1}^{k+1} l \bar{\varphi}^{k+1-l}\left(\bar{\varphi}^{l-1} w\right)_{\dot{x}}+\sum_{l=1}^{k}(k+1-l) \bar{\varphi}^{k-l}\left(\bar{\varphi}^{l}\right)_{\dot{x}} w
$$

The solvability of the described above algebraic systems is obvious for sufficiently small $\tau / h^{3}$. Next let us note that to estimate $\|\varphi\|$ we should have uniform in $j$ estimates for $\left\|y_{x x}^{j}\right\|$. For this reason we consider the $\mathrm{KdV}$ case and use the estimate (44). A detailed analysis ([5]) concludes that the $\varphi$-sequence converges very rapidly,

$$
\frac{c}{\varepsilon}\|\varphi(s+1)-\varphi(s)\|_{(2, \varepsilon)}^{2} \leq\left(\sqrt{\frac{c}{\varepsilon}} \tau\right)^{2^{s}}, \quad s \geq 1,
$$

where $\|f\|_{(r, \varepsilon)}^{2} \stackrel{\text { def }}{=}\|f\|^{2}+\left\|\left(\varepsilon \partial_{x}\right)^{r} f\right\|^{2}$. This implies the statement:

Theorem 6. Let the assumption the Theorem 5 be satisfied. Then the sequence $\varphi(s)$ converges in the $H_{0}^{2}$ sense to the solution of the Equations (52), (57). Moreover,

$$
\|y-\varphi(2)\| \leq c \tau^{2} / \sqrt{\varepsilon},
$$

where $c>0$ does not depend on $h, \tau$, and $\varepsilon$.

The convergence $\varphi(s) \rightarrow y^{j}$ as $s \rightarrow \infty$ for the scheme (61), (62) has been proved numerically. Moreover, it turns out that $\varphi(2)$ approximates the solution $y^{j}$ of (52) sufficiently well. This implies that we set $y^{j}=\varphi(2)$ in the general case also. 
To solve the systems of linear equations of the form (61), (63) and (65), we use the Gauss method adapted to systems with five non-zero diagonals. This implies the efficiency of the schemes in the sense that it executes $O(N)$ arithmetic operations to pass to the next time-level.

To define the function $\omega=\omega(\eta, A)$ and subsequently the initial data (17), we consider the problem

$$
\begin{aligned}
& \frac{d \omega}{d \eta}=-\omega \sqrt{1-g_{1}(A \omega) / g_{1}(A)}, \quad \eta>h, \\
& \omega(h, A)=1-A \frac{h^{2}}{4} \frac{g_{1}^{\prime}(A)}{g_{1}(A)}+A^{2} \frac{h^{4}}{4 !} \frac{g_{1}^{\prime}(A)}{g_{1}^{2}(A)}\left\{g_{1}^{\prime}(A)+\frac{A}{4} g_{1}^{\prime \prime}(A)\right\},
\end{aligned}
$$

and apply the Runge-Kutta method of the fourth order. Next we define $\omega(0, A)=1$ and $\omega(-\eta, A)=\omega(\eta, A)$.

Some results of the numerical simulations are presented in the Figures 1 and 2. The computations have been realized for $\varepsilon=0.1$, $\tau=h^{2}$, and $h=1.7 \cdot 10^{-3}$.

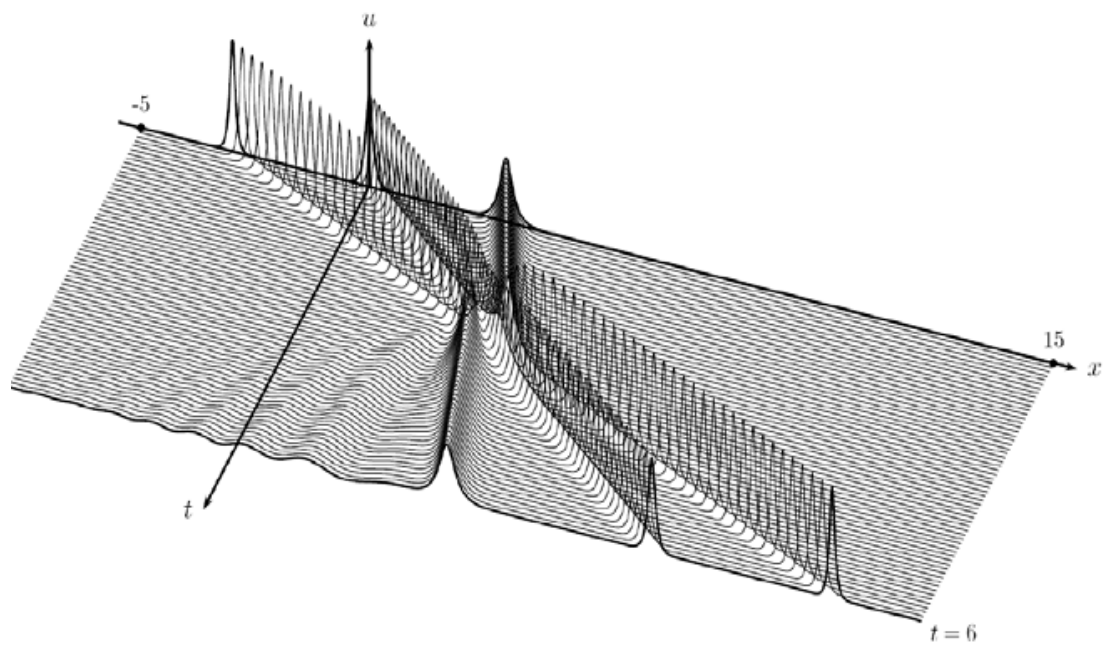

Figure 2. Evolution of the soliton triplet for $g^{\prime}=u^{4}$. 
This numerical scheme is the natural generalization of the algorithms suggested in [5, 6] and [10] for the GKdV equations with homogeneous nonlinearities $g^{\prime}(u)=u^{n}$ and $g^{\prime}(u)=u^{\kappa}$, respectively. The reader can find there all detail of the scheme analysis.

\section{Acknowledgement}

The research was supported by SEP-CONACYT under grant 178690 (Mexico).

\section{References}

[1] E. Abreu, M. Colombeau and E. Panov, Weak asymptotic methods for scalar equations and systems, J. Math. Anal. Appl. 444(2) (2016), 1203-1232.

[2] V. Danilov and D. Mitrovic, Shock wave formation process for a multidimensional scalar conservation law, Quart. Appl. Math. 69(4) (2011), 613-634.

[3] V. G. Danilov and G. A. Omel'yanov, Weak asymptotics method and the interaction of infinitely narrow delta-solitons, Nonlinear Anal. Theory Methods Appl. 54 (2003), 773-799.

[4] V. G. Danilov, G. A. Omel'yanov and V. M. Shelkovich, Weak asymptotics method and interaction of nonlinear waves in: M. V. Karasev ed., Asymptotic methods for wave and quantum problems, AMS Trans., Ser. 2, 208, AMS, Providence, RI, 2003, 33-164.

[5] M. G. Garcia Alvarado and G. A. Omel'yanov, Interaction of solitary waves for the generalized KdV equation, Commun. Nonlinear Sci. Numer. Simul. 17(8) (2012), 3204-3218.

[6] M. G. Garcia Alvarado and G. A. Omel'yanov, Interaction of solitons and the effect of radiation for the generalized KdV equation, Commun. Nonlinear Sci. Numer. Simul. 19(8) (2014), 2724-2733.

[7] H. Kalisch and D. Mitrovic, Singular solutions of a fully nonlinear $2 \times 2$ system of conservation laws, Proc. Edinb. Math. Soc. 55(3) (2012), 711-729.

[8] Kimiaki Konno and Yoshi H. Ichikawa, A modified Korteweg-de Vries equation for ion acoustic waves, J. Phys. Soc. Jpn. 37 (1974), 1631-1636.

[9] G. A. Omel'yanov, Propagation and interaction of solitons for nonintegrable equations, Russ. J. Math. Phys. 23(2) (2016), 225-243. 
[10] G. A. Omel'yanov and M. A. Valdez-Grijalva, Asymptotics for a $C^{1}$-version of the KdV equation, Nonl. Phen. Compl. Sys. 17(2) (2014), 106-115.

[11] O. Rahman, M. D. I. Bhuyan, M. M. Haider and J. Islam, Dust-acoustic solitary waves in an unmagnetized dusty plasma with arbitrarily charged dust fluid and trapped ion distribution, IJAA 4(1) (2014); DOI: 10.4236/ijaa.2014.41011.

[12] H. Schamel, A modified Korteweg-de Vries equation for ion acoustic waves due to resonant electrons, J. Plasma Phys. 9 (1973), 377-387. 